\title{
Kinetic Modeling of Lime-Enhanced Biomass Steam Gasification in a Dual
}

\section{Fluidized Bed Reactor}

\author{
Bijan Hejazi, ${ }^{*}$ John R. Grace, ${ }^{b}$ and Andrés Mahecha-Botero ${ }^{b, c}$ \\ a Chemical Engineering Department, Faculty of Engineering, Ferdowsi University of \\ Mashhad, Mashhad, Iran, Postal code: 9177948944. \\ ${ }^{b}$ Department of Chemical and Biological Engineering, University of British Columbia, \\ 2360 East Mall, Vancouver, BC, Canada, V6T $1 Z 3$. \\ c Present Address: NORAM Engineering, 200 Granville Street, Suite 1800 Vancouver, \\ BC, Canada, V6C 154.
}

*Corresponding author's email: b.hejazi@ferdowsi.um.ac.ir, Bijanhejazi@gmail.com

Tel: 00989155804299 
Table S1

Properties of wood pellets used in experimental study. ${ }^{14}$

\begin{tabular}{lc}
\hline C (wt\% daf) & 49.0 \\
H (wt\% daf) & 6.52 \\
O (wt\% daf) & 44.05 \\
N (wt\% daf) & 0.12 \\
S (wt\% daf) & $<0.05$ \\
Moisture (wt\%, a.r.) & 9.7 \\
Ash (wt\%) & 0.26 \\
LHV (MJ/kg daf) & 17.12
\end{tabular}




\section{Table S2}

Properties of bed material used in experimental study. ${ }^{14}$

\begin{tabular}{ll}
\hline $\mathrm{CaO}(w t \%)$ & 89.5 \\
$\mathrm{MgO}(\mathrm{wt} \%)$ & 0.8 \\
$\mathrm{SiO}_{2}(\mathrm{wt} \%)$ & 7.9 \\
$\mathrm{Al}_{2} \mathrm{O}_{3}(\mathrm{wt} \%)$ & 0.7 \\
$\mathrm{Fe}_{2} \mathrm{O}_{3}(\mathrm{wt} \%)$ & 0.4 \\
$\mathrm{~K}_{2} \mathrm{O}(\mathrm{wt} \%)$ & 0.3 \\
Density $\left(\mathrm{kg} / \mathrm{m}^{3}\right)$ & 1800 \\
Diameter $(\mu \mathrm{m})$ & $300-600$
\end{tabular}


Table S3

Operating conditions for $20 \mathrm{~kW}_{\text {th }}$ experimental study on variable Ca-looping ratio at IFK. ${ }^{14}$

\begin{tabular}{lccccccc}
\hline Experiment number & 1 & 2 & 3 & 4 & 5 & 6 & 7 \\
\hline Fuel flow rate (kg a.r./h) & 3.3 & 3.4 & 3.6 & 3.9 & 4.0 & 4.0 & 4.1 \\
Steam/Fuel Carbon ratio (mol/mol) & 1.5 & 1.5 & 1.4 & 1.3 & 1.3 & 1.3 & 1.3 \\
Fuel flow rate/ bed weight (kg a.r./h)/ & 1.4 & 1.3 & 1.5 & 1.4 & 1.6 & 1.7 & 2.0 \\
kg & & & & & & & \\
Ca/Fuel Carbon (mol/mol) & 7.6 & 7.4 & 1.9 & 11.5 & 4.7 & 2.7 & 0.0 \\
Mean solids residence time (min) & 2.5 & 2.5 & 9.3 & 1.5 & 3.5 & 6.1 & $\infty$ \\
Fuel flow rate/ bed cross-sectional & 326 & 332 & 351 & 379 & 389 & 393 & 397 \\
area & & & & & & & \\
(kg a.r./h)/m² & & & & & & &
\end{tabular}




\section{Table S4}

Operating conditions for $200 \mathrm{~kW}_{\text {th }}$ experimental study on variable gasifier temperature at IFK. ${ }^{16}$

\begin{tabular}{|c|c|c|c|c|c|c|}
\hline Gasifier & Fuel & Steam/Fue & Fuel flow & Ca/Fuel & Mean & Fuel flow \\
\hline temperatur & flow & I Carbon & rate/bed & Carbon & solids & rate/ bed \\
\hline \multirow[t]{3}{*}{ e $\left({ }^{\circ} \mathrm{C}\right)$} & rate & ratio & weight & $(\mathrm{mol} / \mathrm{mol})$ & residence & cross- \\
\hline & $(\mathrm{kg}$ & $(\mathrm{mol} / \mathrm{mol})$ & (kg a.r./h)/ & & time & section $(\mathrm{kg}$ \\
\hline & a.r./h) & & $\mathrm{kg}$ & & (min) & a.r./h) $/ \mathrm{m}^{2}$ \\
\hline $609-756$ & 29.7 & 2.21 & 0.71 & 6.2 & 5.35 & 326 \\
\hline
\end{tabular}




\section{Table S5}

Operating conditions for $200 \mathrm{~kW}_{\text {th }}$ experimental study on variable steam to biomass ratio at IFK.16

\begin{tabular}{|c|c|c|c|c|c|c|c|c|c|}
\hline Experiment number & 1 & 2 & 3 & 4 & 5 & 6 & 7 & 8 & 9 \\
\hline \multirow{3}{*}{$\begin{array}{l}\text { Temperature }\left({ }^{\circ} \mathrm{C}\right) \\
\text { Fuel flow rate }(\mathrm{kg} \text { a.r./h) }\end{array}$} & 679 & 675 & 673 & 683 & 678 & 676 & 675 & 672 & 670 \\
\hline & & & & & & & & & 29. \\
\hline & 29.0 & 29.0 & 29.3 & 29.6 & 29.2 & 29.3 & 29.8 & 29.9 & 5 \\
\hline Steam/Fuel Carbon ratio & 1.61 & 1.64 & 1.92 & 2.22 & 2.25 & 2.55 & 2.80 & 2.78 & 2.8 \\
\hline$(\mathrm{mol} / \mathrm{mol})$ & & & & & & & & & 3 \\
\hline Fuel flow rate/ bed & 0.7 & 0.71 & 0.62 & 0.67 & 0.57 & 0.58 & 0.73 & 0.76 & 0.6 \\
\hline weight & & & & & & & & & 0 \\
\hline (kg a.r./h)/ kg & & & & & & & & & \\
\hline $\mathrm{Ca} /$ Fuel Carbon & & & & & & & & & \\
\hline$(\mathrm{mol} / \mathrm{mol})$ & 5.4 & 5.1 & 4.9 & 5.6 & 5.2 & 5.1 & 4.9 & 4.7 & 4.6 \\
\hline Mean solids residence & 6.2 & 6.5 & 7.8 & 6.3 & 8.0 & 8.0 & 6.6 & 6.6 & 8.5 \\
\hline time (min) & & & & & & & & & \\
\hline Fuel flow rate/ bed cross- & 305 & 305 & 308 & 312 & 307 & 308 & 314 & 314 & 310 \\
\hline sectional area $(\mathrm{kg}$ & & & & & & & & & \\
\hline a.r. $/ \mathrm{h}) / \mathrm{m}^{2}$ & & & & & & & & & \\
\hline
\end{tabular}

